\title{
Seminare / Séminaires / Seminari
}

\section{Praxiseröffnung/-übernahme}

Das Seminar richtet sich an Ärztinnen und Ärzte, die vor einer Praxiseröffnung (Einzel-/ Gruppenpraxis), dem Einstieg in eine Gruppenpraxis oder vor einer Praxisübernahme stehen.

\section{Themen}

- Juristische Aspekte (Praxisbewilligung, Zulassung zur Sozialversicherung, Vertragswesen)

- Gesellschaftsformen / Ehe- und Erbrecht (Privat-/Geschäftsvermögen, Güterstand, Erbschaftsplanung)

- Praxiseinrichtung (Inneneinrichtung, Kostenberechnung)

- Praxisadministration(Leistungserfassungsund Abrechnungssysteme)

- Bewertung einer Arztpraxis (Berechnung Inventarwert und Goodwill)

- Finanzierung der Arztpraxis (Businessplan, Kredite, Absicherungsmöglichkeiten)

- Versicherungen/Vorsorge/Vermögen (Personen- und Sachversicherungen, Vorsorgeplanung)

\section{Sponsoren}

Die Kosten werden durch diverse Sponsoren (siehe www.fmhservices.ch) gedeckt.

\begin{tabular}{lll}
\hline Daten & & \\
\hline K04 & Donnerstag, & Zürich \\
& 3. September 2015 & Volkshaus \\
K05.00-16.30 Uhr & \\
K05 & Donnerstag, \\
& 5. November 2015 & Basel \\
& 09.00-16.30 Uhr & \\
&
\end{tabular}

\section{Praxisübergabe}

Das Seminar richtet sich an Ärztinnen und Ärzte, die ihre Praxis übergeben wollen. Idealtermin: 5-10 Jahre vor geplanter Übergabe (aus steuertechnischen und vorsorgeplanerischen Gründen).

\section{Themen}

- Juristische Aspekte (Praxisübergabevertrag, allg. Vertragswesen, Übergabe der Krankengeschichten)

- Nachfolgeplanung und Bewertung einer Arztpraxis (projektorientiertes Vorgehen in der Nachfolgeplanung, Berechnung Inventarwert und Goodwill als Verhandlungsbasis)

- Versicherungen/Vorsorge/Vermögen (Übergabe/Auflösung von Versicherungsverträgen, Pensions- und Finanzplanung)

- Steuern (Steueraspekte bei der Praxisübergabe: Optimierung der steuerlichen Auswirkungen, Liquidations- und Grundstückgewinnsteuer, Bestimmung des optimalen Übergabezeitpunktes)

\section{Sponsoren}

Die Kosten werden durch diverse Sponsoren (siehe www.fmhservices.ch) gedeckt.

\begin{tabular}{lll}
\hline Daten & & \\
\hline K09 & $\begin{array}{l}\text { Donnerstag, } \\
\text { 10. September 2015 }\end{array}$ & $\begin{array}{l}\text { Zürich } \\
\text { Volkshaus }\end{array}$ \\
K10 & $\begin{array}{l}\text { 13.30-18.00 Uhr } \\
\text { Donnerstag, } \\
\text { 12. November 2015 } \\
\text { 13.30-18.00 Uhr }\end{array}$ & Basel \\
& & \\
\end{tabular}

Finanz- und Steuerplanung

Das Seminar richtet sich an Ärztinnen und Ärzte, die vor einer Praxiseröffnung oder Praxisübernahme stehen oder bereits praxistätig sind.

\section{Themen}

- Finanzplanung (Businessplan, buchhalterische Massnahmen vor Praxiseröffnung/ -übernahme, Standardkontenplan, doppelte Buchhaltung, EDV-unterstützte Buchführungslösung)

- Steuern (Steueraspekte bei Eintritt in die Selbständigkeit, Steuerfallen und Steuerrisiken, optimierte Steuerplanung)

\section{Kosten}

Für FMH Services-Mitglieder kostenlos.

\begin{tabular}{lll}
\hline Daten & & \\
\hline K12 & $\begin{array}{l}\text { Donnerstag, } \\
\text { 17. September 2015 } \\
\text { 13.30-18.00 Uhr }\end{array}$ & Bern \\
& & \\
& & \\
\end{tabular}

\section{Praxiscomputer-Workshop}

Der Workshop richtet sich an Ärztinnen und Ärzte, die vor einer Praxiseröffnung oder Praxisübernahme stehen oder bereits praxistätig sind.

\section{Themen}

- Anforderungen an ein Praxisinformationssystem (Einführung)

- Evaluationsprozess (projektorientiertes Vorgehen in der Evaluation eines Praxisinformationssystems)

- Präsentation von sechs führenden Praxisinformationssystemen (Leistungserfassung, elektronisches Abrechnen unter Einbezug der TrustCenter, Agendaführung, Statistiken, Laborgeräteeinbindung, elektronische Krankengeschichte, Finanzbuchhaltungslösungen usw.)

\begin{tabular}{lll}
\hline Daten & & \\
\hline K15 & Donnerstag, & Olten \\
& 26. November 2015 & Stadttheater \\
& 13.30-18.00 Uhr &
\end{tabular}

Ouverture et reprise d'un cabinet médical

Le séminaire est destiné aux médecins sur le point d'ouvrir un cabinet médical (individuel ou de groupe), de joindre un cabinet de groupe ou de reprendre un cabinet existant.

\section{Contenu}

- Business plan (préparation du plan de financement et crédit d'exploitation, financement par la banque)

- Aménagement (implantation, projet et concept d'aménagement, choix du mobilier, budget)

- Estimation d'un cabinet (inventaire et goodwill)

- Laboratoire

- Administration d'un cabinet médical

- Assurances

- Passage du statut de salarié à celui d'indépendant

- Fiscalité

\section{Sponsors}

Les coûts sont pris en charge par divers sponsors (voir www.fmhservices.ch).

\begin{tabular}{lll}
\hline Dates & & \\
\hline K22 & Jeudi & Lausanne \\
& 3 septembre 2015 & World Trade \\
17h00-21h30 & Center \\
K23 & $\begin{array}{l}\text { Jeudi } \\
\text { 19 novembre 2015 } \\
\text { 13h30-18h00 }\end{array}$ & Genève \\
&
\end{tabular}

Remise d'un cabinet médical

Le séminaire s'adresse aux médecins désirant remettre un cabinet médical. Idéalement 5-10 ans avant la remise prévue (pour des questions de taxation et prévoyance).

\section{Contenu}

- Aspects juridiques (autour du contrat de remise/reprise)

- Estimation d'un cabinet (inventaire et goodwill)

- Assurances (prévoyance, assurances à l'intérieur et autour du cabinet)

- Conséquences fiscales d'une remise

\section{Sponsors}

Les coûts sont pris en charge par divers sponsors (voir www.fmhservices.ch).

\begin{tabular}{lll}
\hline Dates & & \\
\hline K25 & Jeudi & Genève \\
& 12 novembre 2015 & Crowne Plaza
\end{tabular}

$13 \mathrm{~h} 30-18 \mathrm{~h} 00$ 


\section{Apertura e rilevamento di uno studio medico}

Il seminario è destinato ai medici in procinto di aprire o di rilevare uno studio medico.

\section{Contenuto}

- Business Plan (preparazione del piano di finanziamento e del credito d'esercizio, prestito bancario)

- Pianificazione (insediamento, progetto e pianificazione, scelta del mobilio, budget)

- Valutazione di uno studio medico (inventario e goodwill)

- Amministrazione di uno studio medico (interna allo studio, rapporti con la banca)

- Assicurazioni (tutte le assicurazioni necessarie interne ed esterne allo studio)

- Passaggio dallo stato di dipendente a quello di indipendente

- Fiscalità
Sponsor

Diversi sponsor si fanno carico delle spese (si rimanda al sito www.fmhservices.ch).

\begin{tabular}{lll}
\hline Date & & \\
\hline K51 & Giovedì & Chiasso \\
& $\begin{array}{l}\text { 22 ottobre } 2015 \\
\text { dalle } 14.00 \text { alle } 17.30\end{array}$ & FMH Consulting \\
& Services
\end{tabular}

Anmeldung und Auskunft /

Inscription et information /

Iscrizioni e informazioni

www.fmhservices.ch oder FMH Consulting Services, Cornelia Fuchs, Tel. 0419250077

\section{Hinweis / Remarque / Osservazioni}

Bei sämtlichen Seminaren, bei denen die Kosten teilweise oder gänzlich von Seminarsponsoren gedeckt werden, werden die Teilnehmeradressen den jeweiligen Sponsoren zur Verfügung gestellt.

Les adresses des participants aux séminaires dont les coûts sont couverts en partie ou totale- ment par des sponsors sont communiquées aux sponsors concernés.

Gli indirizzi dei partecipanti ai seminari, i cui costi sono coperti in parte o completamente da degli sponsor, vengono comunicati agli sponsor interessati.

Annullierungsbedingungen / Conditions d'annulation / Condizioni d'annullamento Bei Abmeldungen oder Fernbleiben werden folgende Unkostenbeiträge erhoben:

Un montant est perçu pour une absence ou une annulation. Il est de:

Un importo verrà rimborsato in caso di assenza o annullamento. Esso sarà di:

- 50 CHF pro Person ab 14 Tage vor Seminarbeginn / par personne dans les 15 jours avant le début du séminaire / per persona entro i 15 giorni prima dell'inizio del seminario;

- 100 CHF pro Person ab 7 Tage vor Seminarbeginn oder Fernbleiben / par personne dans les 7 jours avant le début du séminaire / per persona entro i 7 giorni prima dell'inizio del seminario.

\section{Seminarsponsoren 2015}

Die Unterstützung durch verschiedene Sponsoren ermöglicht es der FMH Consulting Services AG, ihre Seminarreihen für FMH Services-Mitglieder teils kostenlos, teils kostengünstig anzubieten. Gerne stellen wir Ihnen diese Firmen in einem Kurzporträt vor.

\section{medics labor}

professionell und persönlich

Medics Labor AG

Chutzenstrasse 24, 3001 Bern

Tel. 03137220 02, Fax 0313714044

info[at]medics-labor.ch

www.medics-labor.ch

\section{Medizinisches Labor und mehr}

Medics Labor ist ein Schweizer Unternehmen, zu Hause in Bern, hier verwurzelt und seit vielen Jahren erfolgreich tätig im Kanton sowie weiteren Regionen.

Geschätzt als persönliches, unkompliziertes Gegenüber, überzeugt Medics Labor durch fachliches und menschliches Gespür mit zahlreichen Hilfestellungen und Dienstleistungen. Wir verstehen uns als sozialer Arbeitgeber und beschäftigen auch behinderte Personen.

Medics Labor ist ein Labor von Ärzten für Ärzte. Es gehört den Laborspezialisten und den Ärzten, die das Unternehmen gemeinsam führen.

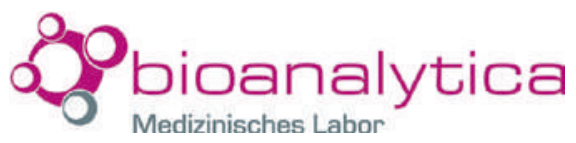

Bioanalytica AG

Maihofstrasse 95a, 6006 Luzern

Tel. 041429 31 31, Fax 0414293130

service[at]bioanalytica.ch

www.bioanalytica.ch

\section{Engagierte Kompetenz}

Bioanalytica, 1957 in Luzern gegründet, basiert auf einer langjährigen Tradition. Stetige Innovation und ein Team qualifizierter Fachspezialisten und Labormediziner bilden das Fundament unserer Kompetenz. Qualität und Seriosität das sind die Werte, denen wir uns verschrieben haben. Aus der Überzeugung, dass dies auch unseren Kunden wesentliche Vorteile bietet, haben wir unser Labor im Jahre 2000 akkreditieren lassen.

Schnell, wenn notwendig auch rund um die Uhr, sind wir für Sie da. Mit dem Know-how von rund 85 Mitarbeitenden, modernster Laborautomation und Informationstechnologie sind unsere Laborresultate in kürzester Zeit verfügbar. Bei Bioanalytica stehen Sie als Kunde im Mittelpunkt. Wir unterstützen Sie und Ihr Praxisteam jederzeit gerne optimal im persönlichen Kontakt und mit zahlreichen wertvollen zusätzlichen Dienstleistungen.

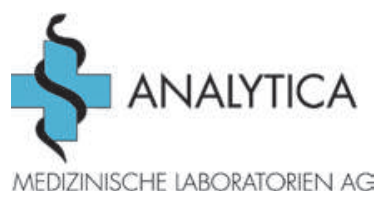

Analytica Medizinische Laboratorien AG Falkenstrasse 14 8024 Zürich

Tel. 04425050 50, Fax 0442505051

kundendienst[at]analytica.ch

www.analytica.ch

Werte. Verbinden.

Sehr geehrte Frau Kollega, sehr geehrter Herr Kollege

Die Analytica Medizinische Laboratorien AG wurde 1957 von meinem Vater gegründet und ich durfte das Labor 1985 in zweiter Generation übernehmen. Eigentlich mag ich es gar nicht, mich unpersönlich und mit schönen Worten vorzustellen. Ich bin durch und durch Praktiker, gibt es ein Problem, dann löse ich es und kennt mich ein Kunde noch nicht persönlich, dann komme ich gerne vorbei und stelle mich vor. Wir haben uns nach langen Diskussionen für den Slogan «Werte. Verbinden.» entschieden. Der Punkt zwischen den Worten ist kein Schreibfehler. Wir haben Werte. Werte, die uns einen persönlichen Umgang miteinander ermöglichen, Werte, die die Qualität unserer Arbeit beschreiben und Werte, die wir mit Ihnen unseren Kunden - teilen. Diese Werte verbinden uns miteinander und stellen das tragfähige Netz dar, das uns alle seit so vielen Jahren trägt. Für das bin ich dankbar.

Dr. med. Peter Isler

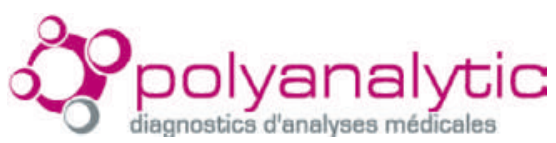

Polyanalytic SA

Avenue de Sévelin 18, 1004 Lausanne

Tel. 02180492 50, Fax 0218049250

info[at]polyanalytic.ch

www.polyanalytic.ch

Polyanalytic ist ein Labor für medizinische Analysen, das auf dem Gebiet der Kantone Waadt und Neuenburg tätig ist.

Gestützt auf seine Kompetenzen, die es in den Dienst der Patientinnen und Patienten und der Ärzteschaft stellt, bietet Polyanalytic eine umfassende Palette von medizinischen Analysen. Seit Polyanalytic 1983 gegründet wurde, ist das Unternehmen für herausragende Qualität und kundennahe Dienstleistungen bekannt. Den frei praktizierenden Ärztinnen und Ärzten werden mit unvergleichlicher Konstanz verlässliche, rasche und kompetente Leistungen geboten, damit sie ihre Kunst ausüben können. 
Mit Polyanalytic verfügen die Ärztinnen und Ärzte nicht nur über einen Partner, der auf ihre Bedürfnisse eingeht, sondern auch tagtäglich über echte Unterstützung bei ihrer Tätigkeit. Polyanalytic ist mehr als ein Unternehmen: Dank der Kompetenz der Menschen, die dort arbeiten, kann die Ärzteschaft darauf vertrauen, dass bei den Patientinnen und Patienten, für die sie verantwortlich ist, optimale Laborkontrollen gewährleistet sind.

\section{¿ु్j dianalabs}

Dianalabs SA

Rue de la Colline 6, 1205 Genève

Tel. 02280712 40, Fax 0228071244

info[at]dianalabs.ch

www.dianalabs.ch

Dianalabs ist ein Labor für medizinische Analysen, das 1988 mit dem Ziel gegründet wurde, der Ärzteschaft und den Patientinnen und Patienten optimale Laborkontrollen zu bieten.

Aufgrund seiner Publikationen und Präsentationen ist das Genfer Labor für die Qualität seiner Serologie international anerkannt.

Wir bieten eine umfassende Palette von medizinischen Analysen, um alle Bedürfnisse der Medizin abzudecken. Doch Dianalabs ist mehr als ein polyvalentes Allround-Labor: Dank seinem Spezialistenteam deckt es eine Vielzahl von Fachgebieten ab und bietet eine Schnittstelle zu jedem medizinischen Fachgebiet mit seinen besonderen Bedürfnissen.

Durch die wissenschaftliche Zusammenarbeit mit den Ärztinnen und Ärzten und den universitären Zentren wurde uns klar, dass nur ein regionales Unternehmen, das grundlegende menschliche Werte wie Qualität, Austausch und Dienstleistungsbereitschaft in den Vordergrund stellt und mit den lokalen Verhältnissen vertraut ist, die Bedürfnisse der Bevölkerung und der Ärzteschaft kompetent erfüllen kann.

\section{Schmid} MOGELSBERG

MEDIPRINT. CLASSICPRINT

Schmid Mogelsberg AG

Sonnmattstrasse 1, 9122 Mogelsberg

Tel. 07137560 80, Fax 0713756081

info[at]schmid-mogelsberg.ch

www.schmid-mogelsberg.ch

\section{Ärztedrucksachen - 80 Jahre im Dienste der Ärzte}

Seit 80 Jahren pflegen wir die Kunst des Details. Deshalb vertrauen uns im Bereich Mediprint über 9000 Ärzte in der ganzen Schweiz. Qualität, Perfektion und Vertrauensbildung sind für uns ebenso ausschlaggebend wie für Sie als Kunde. Nehmen Sie unsere sorgfältige Beratung oder unsere Druckmusterkollektion in Anspruch. Anruf genügt.

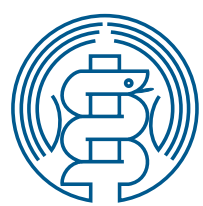

Schweizerische Ärzte-Krankenkasse Oberer Graben 37, Postfach 2046 , 9001 St. Gallen

Tel. 07122718 18, Fax 0712271828

info[at]saekk.ch / www.saekk.ch

Die richtige Adresse für Erwerbsausfalldeckungen, Kollektivkrankenkasse und Versicherungsplanung.

Mit mehr als 110 Jahren Erfahrung kennt unsere Organisation auch heute die Bedürfnisse der Ärztinnen und Ärzte. Sie bietet entsprechend durchdachte und kostengünstige Lösungen an, sowohl für Praxiseröffner/-innen wie auch für selbständige und angestellte Ärztinnen und Ärzte.

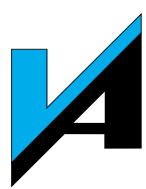

Versicherung der Schweizer Ärzte Genossenschaft

Länggassstrasse 8, 3000 Bern 9

Tel. 03130125 55, Fax 0313025156

versa[at]versa.ch / www.versa.ch

Spezialisiert auf die Bedürfnisse von Ärztinnen und Ärzten und deren Ehegatten, bietet die Versicherung der Schweizer Ärzte Genossenschaft individuelle, flexible und kostenoptimierte Lösungen für Risikoabdeckungen (Tod und Invalidität) sowie für den sicheren Kapitalaufbau im Bereich der privaten Vorsorge an.

\section{Kearchiv-schweiz}

kgarchîv-schweiz

Berner Archiv AG

Bernstrasse 23, 3122 Kehrsatz

Tel. 0319601070

info[at]kgarchiv.ch / www.kgarchiv.ch

kgarchîv-schweiz bietet in Zusammenarbeit mit FMH Services datenschutzkonforme Systeme, um Ärzte/-innen die Archivierung der Krankengeschichten gemäss Standesregeln abzunehmen.

Wir übernehmen sämtliche Archivaufgaben nach der Praxisaufgabe. Wir bieten mit Scanning on demand eine einfache und kostengünstige Lösung bei der Umstellung von der physischen zur digitalen Krankengeschichte an.

Das Krankengeschichten-Archiv ist eine spezifische Dienstleistung der Berner Archiv AG. Die sehr sensiblen Patientendaten verlangen nach einem sorgfältigen, fachgerechten und datenschutzkonformen Handling. Entsprechend zertifiziertes Fachpersonal erfüllt diese Aufgabe gemäss den ISO-Normen 9001/2008, Goodpriv@cy+VDSZ Datenmanagement.

\section{medica MEDIZINISCHE LABORATORIEN Dr. F. KAEPPELI AG}

MEDIZINISCHE LABORATORIEN

DR. F. KAEPPELI AG

Eidgenössisch anerkannte Laboratorien Wolfbachstrasse 17, 8024 Zürich

Tel. 04426999 99, Fax 0442699909 info[at]medica-labor.ch

www.medica-labor.ch

Der promovierte Mikrobiologe und Biochemiker Dr. F. Käppeli, Laborspezialist FAMH, übernahm 1976 das heute über 50-jährige Unternehmen und gründete als dessen Leiter und Inhaber die Einzelfirma medica. Der wichtigste unternehmerische Leitgedanke von Dr. F. Käppeli heisst kontinuierliche Innovation und Schaffung wegweisender Standards auf allen Gebieten der Labormedizin: Mikrobiologie inklusive Parasitologie, Serologie, Immunologie, klinische Chemie, Hämatologie, molekulare Diagnostik und Pathologie in Human- und Veterinärmedizin. So entstand ein Kompetenz-Zentrum für Labordiagnostik von gesamtschweizerisch grosser Bedeutung. Die modernst ausgebauten Laboratorien werden laufend erweitert und befinden sich im Herzen von Zürich. Über 200 Angestellte der Partnerlabors, begleitet von Spezialisten aus Medizin, Pharmakologie, Naturwissenschaften und Technik, garantieren für höchste Professionalität.

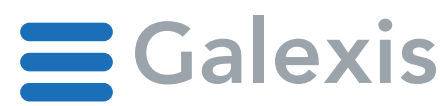

Galexis AG

Industriestrasse 2, Postfach, 4704 Niederbipp Tel. 0588517111 , Fax 0588517114 info[at]galexis.com / www.galexis.com

Als Vollgrossist setzt Galexis AG Standards im Schweizer Gesundheitsmarkt. Wir beliefern unsere Kunden ganz nach dem Motto "Alles aus einer Hand» mit Pharma, Praxis- und Laborbedarf sowie Medizintechnik und erbringen darüber hinaus integrierte Dienstleistungen in der Gesundheitslogistik - schweizweit. Mit erprobten Lösungen fördert Galexis den Erfolg ihrer Kunden.

Möchten Sie ausserdem Ihre eigene Praxis praktisch, funktionell und ästhetisch einrichten? Genau hier kann Sie Galexis mit ihren Fachpartnern und einer langjährigen Erfahrung professionell beraten und unterstützen! Überzeugen Sie sich - mit Galexis können Sie rechnen!

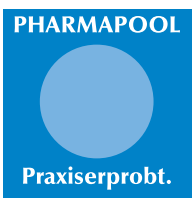

Pharmapool AG

Unterlettenstrasse 18, 9443 Widnau

Tel. 07172725 25, Fax 0717272555

info[at]pharmapool.ch / www.pharmapool.ch 
Pharmapool ist der ärzteeigene Grossist mit 24-Stunden-Lieferbereitschaft. Das Kennen der Bedürfnisse von Arzt und Praxispersonal und das Wissen über die medizinischen Abläufe stehen im Mittelpunkt.

Dank der fundierten Betreuung und rationellen Belieferung wird die Wirtschaftlichkeit der von Pharmapool bedienten Praxen verbessert sowohl von rezeptierenden als auch selbstdispensierenden Ärzten. Unabhängig von Einzelinteressen der Pharma-Branche erhalten unsere Kunden das gesamte Sortiment an Originalprodukten, Generika, Seren, Verbrauchsmaterialien, Einrichtungsgegenständen und MiGeLArtikeln zu transparenten Konditionen.

Eng verbunden mit der attraktiven Lieferpolitik sind unsere praxiserprobten Dienstleistungen rund um die Medikamenten-Logistik, wie z.B. modernes Bestellwesen sowie praktische Preisnachführung und hilfreiche Einkaufs-Statistiken. Pharmapool - von Ärzten für Ärzte.

\section{diatools \\ diagnostics solutions}

Diatools AG

Durisolstrasse 12e, 5612 Villmergen

Tel. 0878800 232, Fax 0878800233

info[at]diatools.ch

www.diatools.ch

\section{Innovative Lösungen im Praxislabor}

Die Diatools AG ist seit dem Jahr 2000 im Bereich Labordiagnostik tätig und hat sich mit zuverlässigen Produkten und kompetenten Dienstleistungen einen Namen gemacht

Dank der langjährigen Erfahrung unserer Mitarbeiter im Diagnostikbereich und einem Netzwerk von ausgesuchten Partnerfirmen bieten wir unseren Kunden auf ihre Bedürfnisse abgestimmte Dienstleistungen an.

Produktpalette: Klinische Chemie (Dialab Autolyser); Elektrolyt Analyser (Medica EasyElectrolytes); Hämatologie (Swelab Alpha); PT/INR (Alere INRatio ${ }^{\circledR} 2$ ); HbA1c/CRP/ACR (Alere Afinion AS100); Urinanalytik (Analyticon Urilyzer ${ }^{\circledR 100) ; ~}$ Schnellteste (Diagnostik Nord).

Legen Sie Wert auf individuelle Betreuung, umfassenden Service und kundenorientierte Lösungen, dann sind Sie bei uns an der richtigen Adresse.

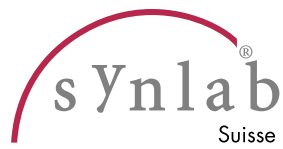

synlab Suisse SA

Alpenquai 14, 6002 Luzern

Tel. 04136035 35, Fax 0413607294

labor.suisse[at]synlab.ch

www.synlab.ch

\section{synlab Suisse - Der Laborpartner}

in Ihrer Nähe

synlab Suisse ist an 20 Standorten, inklusive überregionalen Kompetenzzentren, mit 500 hochqualifizierten Mitarbeitenden in der gesamten Schweiz vertreten. Die Nähe zu unseren
Kunden ist uns wichtig. Dies garantiert eine schnelle Abwicklung, gekoppelt mit fundierter medizinischer Erfahrung, moderner Analysentechnik und Resultate-Übermittlung. Wir bieten unseren Kunden ein umfassendes Angebot an Dienstleistungen, von der Routineanalytik bis hin zu Spezialanalysen, dies umfasst über 4000 verschiedene Analysen aus allen Bereichen der Labormedizin.

synlab - Synergien, die Werte schaffen

synlab ist ausserdem eine der führenden medizinisch-analytischen Laborgruppen in Europa. synlab ist in 23 Ländern vertreten und beschäftigt ca. 7000 Mitarbeiter, die sich täglich für das Wohlergehen der Patienten engagieren.

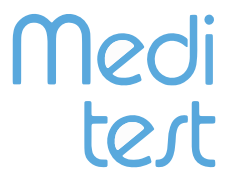

Laboratoire Meditest Vevey SA

Av. Général-Guisan 30B, 1800 Vevey

Tél. 02192540 20, Fax 0219229288

info[at]meditest.ch / www.meditest.ch

Meditest est un laboratoire d'analyses médicales Suisse fondé à Vevey en 1978 dans le but d'offrir un service de proximité à haute valeur ajoutée et des prestations sur mesure aux médecins et aux patients de Romandie. Le groupe Meditest s'est développé dans toute la Suisse Romande depuis 1979 et a depuis 1992 exporté son savoir faire à Singapour.

Depuis maintenant plus de 30 ans, notre philosophie est restée la même, nous associons les plus hautes compétences intellectuelles aux dernières innovations technologiques en matière d'interprétation, de diagnostic, et de prévention. Nous cherchons toujours à nous adjoindre les services de médecins et consultants reconnus dans leurs domaines pour offrir un diagnostic complet, cela toujours dans un esprit de service sur mesure et de proximité.

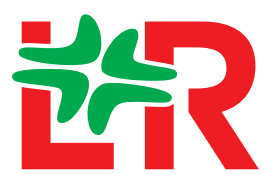

Lohmann \& Rauscher AG

Oberstrasse 222, 9014 St. Gallen

Tel. 07127425 70, Fax 0712742571

info[at]ch.LRmed.com

www.Lohmann-Rauscher.ch

L\&R ist ein international führender Anbieter von erstklassigen Medizin- und Hygieneprodukten höchster Qualität - vom klassischen Verbandstoff bis zum modernen Therapie- und Pflegesystem. 1998 aus den Unternehmen Lohmann (gegründet 1851) und Rauscher (gegründet 1899) entstanden, verfügt L\&R über mehr als 160 Jahre Kompetenz als zuverlässiger Problemlöser für seine Kunden. Mit über 4000 Mitarbeitern, 37 Konzerngesellschaften und Beteiligungen sowie mehr als 130 ausgewählten
Partnern ist L\&R in allen wichtigen Märkten der Welt vertreten und konnte sein Umsatzvolumen 2013 auf 530 Millionen Euro steigern. Das 100-prozentige Tochterunternehmen Lohmann \& Rauscher Schweiz sitzt in St. Gallen und vertreibt einen Grossteil der internationalen L\&R Marken aus den Bereichen Setsysteme \& Hygiene, Unterdrucktherapie, Wundversorgung, Kompressionstherapie sowie Mobilisation und Immobilisation.

\section{SANDOZ}

a Novartis company

Sandoz Pharmaceuticals AG Suurstoffi 14, 6343 Rotkreuz Tel. 04176374 11, Fax 0417637400 info.switzerland[at]sandoz.com www.generika.ch

\section{Sandoz Pharmaceuticals AG}

Sandoz Pharmaceuticals AG ist der führende Generika-Anbieter in der Schweiz. Das Produktportfolio beinhaltet über 180 Wirkstoffe in über 1100 Darreichungsformen und deckt damit viele Indikationsgebiete ab. Zudem zeichnet sich Sandoz durch die Entwicklung und Herstellung von differenzierten Generika und Biosimilars (Folgepräparate patentierter biotechnologischer Arzneimittel) aus. Derzeit sind drei Biosimilars in der Schweiz zugelassen. Sandoz Schweiz versteht sich als vertrauenswürdiger Partner für die Ärzte, Apotheker und Patienten. Umfassende Serviceleistungen wie Patientenbroschüren, klar bezeichnete Verpackungen, benutzerfreundliche und innovative Darreichungsformen runden das Angebot ab.

\section{N NEUROTH Medical}

Neuroth Medical Switzerland AG Allmendstrasse 11, 6312 Steinhausen Tel. 0800100 018, Fax 0417413011 medical[at]neuroth.ch www.neuroth.ch

Ein verlässlicher Partner: Damit Sie sich auf Ihre Patienten konzentrieren können.

Die Neuroth Medical Switzerland AG ist ein eigenständiges Schweizer Unternehmen und Teil der Neuroth-Gruppe, Österreichs ältestes Familienunternehmen für Hörgeräteakustik. Die bereits über 60 Jahre lange Erfahrung der Neuroth Medical hat gezeigt, dass Kunden bzw. Patienten von einer engen, partnerschaftlichen Zusammenarbeit mit ORL-Ärzten profitieren. Die Neuroth Medical Switzerland AG bietet das erlangte Know-how des österreichischen Marktführers in ORL-Medizintechnik in Kombination mit qualitativ hochwertigen Produkten (Zeiss, Storz, Atmos, Interacoustics, Otodynamics, Melag, Miele etc.) nun vor Ort für Sie an: von der Praxisplanung bis zur ORL-Einheit; von der Pinzette bis zum RF-Gerät; vom OAE-Screener bis zur kompletten neurootologischen Testbatterie; vom Desinfektionsmittel bis zum Hygieneplan. 